Check for updates

Cite this: RSC Adv., 2018, 8, 1852

\title{
One-dimensional molecular chains formed by Sierpiński triangles on $\mathrm{Au}(111) \dagger$
}

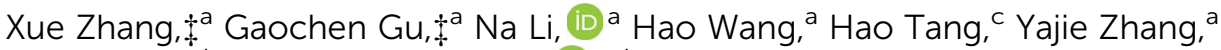 \\ Shimin $\mathrm{Hou}^{\mathrm{ab}}$ and Yongfeng Wang (D) *ab
}

One-dimensional molecular chains with Sierpiński triangles as building blocks were prepared on Au(111) and studied by low-temperature scanning tunneling microscopy. Fractal Sierpiński triangles were stabilized by the coordination interaction between 1,3-bi(4-pyridyl) benzene (BPyB) and Co atoms. At low coverages, Sierpiński triangles of high orders were observed. At high coverages, Sierpiński triangles formed two types of one-dimensional molecular chains. The high coverages and matching between molecular size and surface lattices are attributed to the formation of molecular chains.

Received 26th October 2017

Accepted 21st December 2017

DOI: 10.1039/c7ra11825b

rsc.li/rsc-advances

weak molecule-substrate interactions which allow molecular free diffusion. Secondly, using intermediately strong interactions as driving forces can make sure both the self-correction of defects formed during growth and sufficient molecular diffusion. Thirdly, the structure matching between molecules and surface lattices favors the formation of long-range ordered structures.

Based on above considerations, $120^{\circ}$ V-shaped BPyB molecules and Co atoms are used to build arrays of STs on Au(111). high-order STs or regular patterns with STs as building blocks need to be constructed. Due to the limitation of kinetic growth, it is of high challenge to prepare high-order STs. As shown by the scanning tunneling microscopy (STM) experiments, the largest order of obtained STs is only $5 .{ }^{17}$ Preparation of ST arrays looks promising because various ways have been found to grow ordered patterns on surfaces. By a templating method, onedimensional double chains of STs were prepared along the reconstructed rows on $\mathrm{Au}(100)$ at low molecular coverages. ${ }^{18}$ Without templates, large-scale ordered patterns of STs has not been prepared on $\mathrm{Au}(111)$ using the longer 4,4"-dicyano$1,1^{\prime}: 3^{\prime}, 1^{\prime \prime}$-terphenyl (C3PC) molecule.

Several factors are needed to be taken into account to construct regular crystalline arrays with STs as the building block on the three-fold $\mathrm{Au}(111)$ substrate. Firstly, inert surfaces and molecules with smaller sizes than C3PC are needed to get

\footnotetext{
${ }^{a}$ Key Laboratory for the Physics and Chemistry of Nanodevices, Department of Electronics, Peking University, Beijing 100871, China. E-mail: yongfengwang@pku. edu.cn

${ }^{b}$ Peking University Information Technology Institute, Tianjin Binhai, Tianjin 300450, China

${ }^{c}$ Groupe Matériaux Crystallins sous Contrainte, CEMES-CNRS, Boîte Postale 94347, Toulouse 31055, France

$\dagger$ Electronic supplementary information (ESI) available. See DOI: 10.1039/c7ra11825b

\$ These authors contributed equally to this work.
}

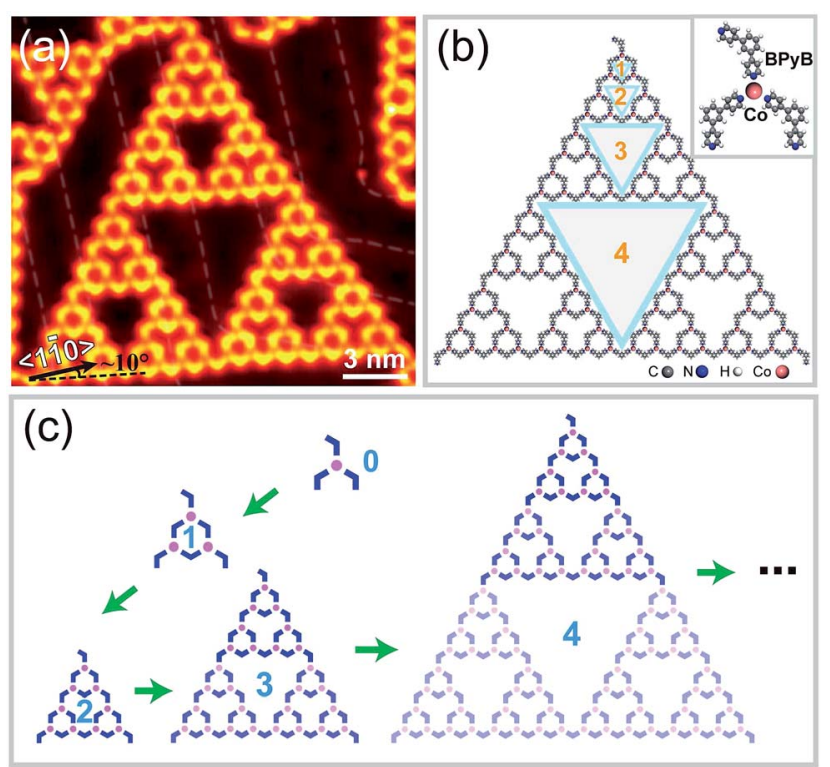

Fig. 1 Fractal phase formed at low molecular coverage. (a) STM image of a defect-free ST of 4 order; inset: coordinated trimer acting as basic recursive unit. The white arrow indicates $\langle 1 \overline{1} 0\rangle$ direction. Scanning parameters: constant-height mode, $V_{\mathrm{B}}=10 \mathrm{mV}, \mathrm{I}_{\mathrm{t}}=80 \mathrm{pA}$. (b) Structural model of the ST in (a). (c) Schematic models revealing the evolution process of fractal phase. 
Low-temperature STM was used to characterize the formed structure at the single-molecule level. At low coverage, STs up to fourth order were observed in measurements. At high coverage, two types of large-scale arrays with STs as the repeated units were successfully prepared. As a comparison, the disordered structure formed by Co atoms and C3PC molecules at high molecular coverages was investigated.

Experiments were performed by a Unisoku scanning tunneling microscope (STM) operating at $4.3 \mathrm{~K}$ with a base pressure of $10^{-10}$ torr. The single-crystalline $\mathrm{Au}(111)$ surface was cleaned by cycles of $\mathrm{Ar}^{+}$sputtering and annealing. The cut $\mathrm{Pt} / \mathrm{Ir}$ tip was first annealed in vacuum chamber and then dipped into $\mathrm{Au}(111)$ in a controlled way. The BPyB and C3PC molecules were thermally deposited onto the substrate from Ta boats, separately. The Co atoms were evaporated from a Knudsen cell onto $\mathrm{Au}(111)$. The ratio between Co and $\mathrm{BPyB}$ was slightly larger than $2: 3$ during deposition. The substrate was kept at room temperature when depositing molecules and Co atoms. All STM images were slightly smoothed using software WSxM. ${ }^{19}$

After sublimating BPyB molecules and Co atoms onto $\mathrm{Au}(111)$ and annealing at $400 \mathrm{~K}$ for 10 minutes, isolated metalorganic coordinating STs were obtained in experiments at low molecular coverages. Here we denote STs of order $n$ as BPyB-Co-
ST- $n$ or ST- $n$, where $n$ ranges from 0 to 4 . Fig. 1(a) and (b) present a BPyB-Co-ST-4 and its chemical structure, respectively. The node in STs is formed by one Co atom and three BPyB molecules through coordinating interaction, as shown in the upper right in Fig. 1(b). There are 81 Co atoms and 123 BPyB molecules in a BPyB-Co-ST-4. The iterative process of ST- $n$ is demonstrated in Fig. 1(c) where the chemical structure of BPyB was simplified to a blue $120^{\circ}$ polyline. In a BPyB-Co-ST- $n$, the number of BPyB molecules $\left(N_{\mathrm{BPyB}}\right)$ and Co atoms $\left(N_{\mathrm{Co}}\right)$ are: $N_{\mathrm{BPyB}}=3\left(3^{n}+1\right) / 2$ and $N_{\text {Co }}=3^{n}$. Close inspection of the BPyB-Co-ST-4 reveals that they contain various pores of different sizes (Fig. 1(b)). The area of largest pore is around $44.6 \mathrm{~nm}^{2}$. When increasing the coverage, BPyB molecules would cover the pores and the order $n$ of STs would decrease.

Fig. 2(a) shows a large-scale STM image of Co-BPyB structures on $\mathrm{Au}(111)$, which was prepared by depositing $\mathrm{BPyB}$ molecules and Co atoms at high coverages and annealing at 400 $\mathrm{K}$ for 10 minutes. Partial STs are marked by triangles to guide the eye. Ordered packing of STs is found in the figure. One representative packing mode of STs is shown in Fig. 2(b). Onedimensional (1D) molecular ribbons consist of pairs of BPyBCo-STs-2 marked by white dashed triangles. The high-resolution STM image and chemical structure are displayed in Fig. 2(c) and
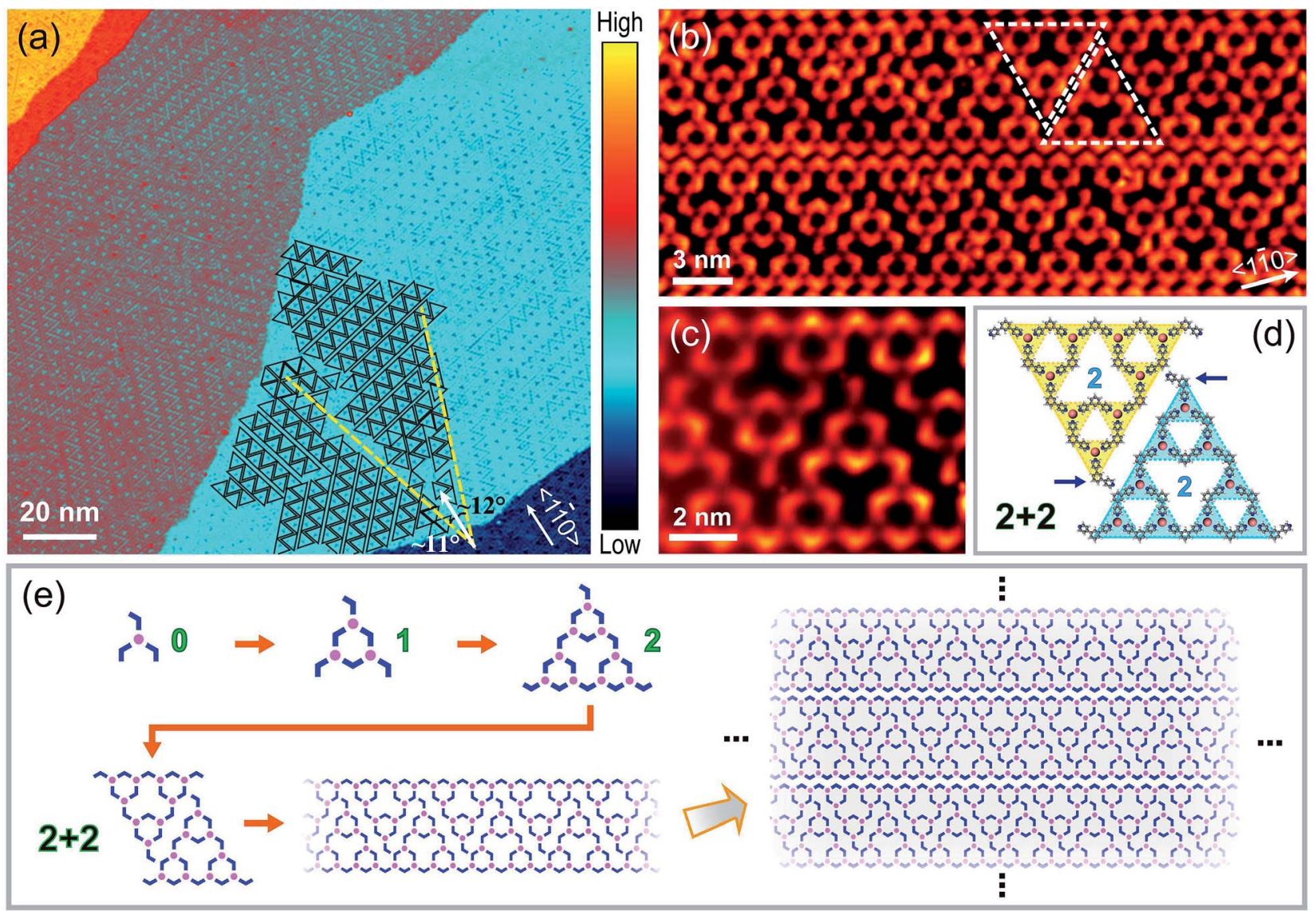

Fig. 2 BPyB-CO-ST-2 arrays formed at high molecular coverage. (a) Large area of molecular ribbons formed by close-packed second order fractals. (b) Close-up image showing four compact rows of 1D molecular ribbons. (c) Details of two interlocking fractals (2 +2 ) playing the role of initial repetitive unit. (d) Structural models corresponding to combination $2+2$. (e) Hierarchical formation of compact 1D molecular ribbons originated from 1.6D fractal moieties. The white arrow points to $\langle 1 \overline{1} 0\rangle$ direction. Scanning parameters: (a) constant-current mode, $V_{B}=1 \mathrm{~V}, I_{\mathrm{t}}=12$ $\mathrm{pA}$; (b) constant-current mode, $V_{\mathrm{B}}=30 \mathrm{mV}, I_{\mathrm{t}}=12 \mathrm{pA}$; (c) constant-height mode, $V_{\mathrm{B}}=50 \mathrm{mV}, I_{\mathrm{t}}=12 \mathrm{pA}$. 
(d). Due to the space limitation, the marked pyridyl groups in apical molecules of STs are partially lifted from the $\mathrm{Au}(111)$ substrate (Fig. 2(d)). As a result, they appear darker than the rest parts of molecules in the STM image (Fig. 2(c)) because of a weaker coupling to the substrate. The molecular models in Fig. 2(e) are used to illustrate the hierarchically self-assembling process of $1 \mathrm{D}$ chains. The combination of a Co atom and three BPyB molecules leads to the formation of a threefold heterotactic node or ST-0. After the iterative process, the ST- 1 and ST-2 can be obtained. Lateral translating the rhombic pair of STs-2 produces 1D molecular chains. Vertical packing molecular chains finally cause the formation of the experimentally observed chain array.

Interestingly, another type of 1D structure was observed when annealing the sample at a lower temperature of $330 \mathrm{~K}$ at high coverages (Fig. 3(a)). These double chains are stabilized by mixed three- and four-fold coordination interactions. The structure marked by white dashed frames was magnified in Fig. 3(b). The high-resolution STM image reveals that the basic repetitive unit contains two BPyB-Co-STs- 1 connected through a fourfold coordination node. The chemical structures are overlaid on the right side of the image to guide the eye. Similar to Fig. 2(e), the scheme in Fig. 3(c) is used to reveals the forming sequence of hierarchical chains shown in Fig. 3(a). Density Functional Theory (DFT, ESI $\dagger$ ) calculations reveal that the average binding energies of $\mathrm{Co}(\mathrm{BPyB})_{3}$ and $\mathrm{Co}(\mathrm{BPyB})_{4}$ are -1.63 and $-1.47 \mathrm{eV}$, respectively. When annealing at $330 \mathrm{~K}$, the insufficient molecular diffusion results in the metastable fourfold coordination. As expected, the surface structure changes
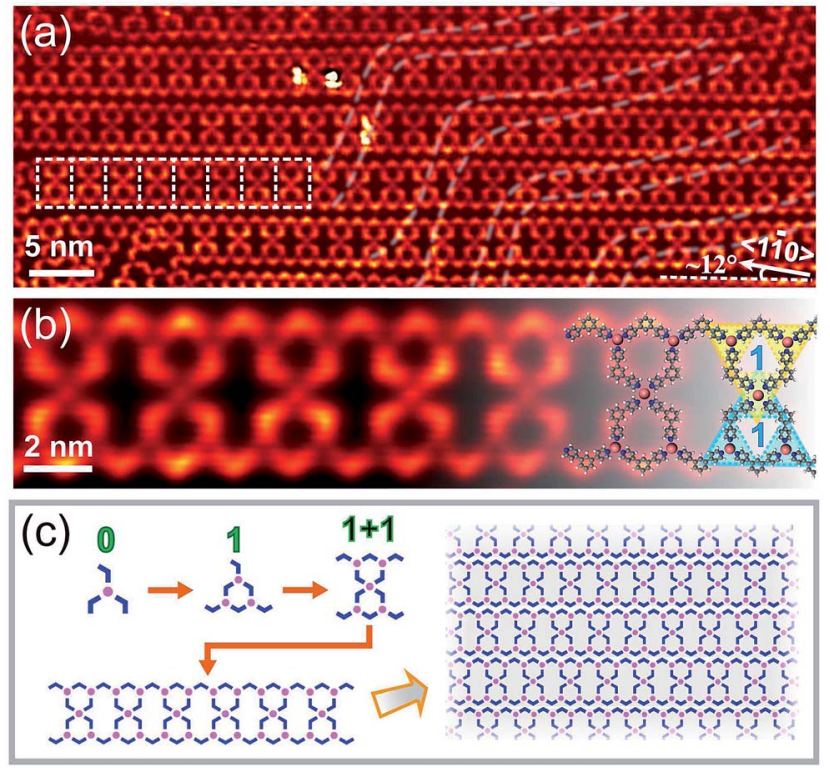

Fig. 3 BPyB-Co-ST-1 arrays formed at high molecular coverage. (a) STM image of 1D molecular ribbons formed by combination of $1+1$. (b) Detailed image of one $1+1$ ribbon superposed by models. The white arrow points to $\langle 1 \overline{1} 0\rangle$ direction. Scanning parameters: constantheight mode, (a) $V_{\mathrm{B}}=50 \mathrm{mV}, \mathrm{I}_{\mathrm{t}}=60 \mathrm{pA}$; (b) $V_{\mathrm{B}}=10 \mathrm{mV}, I_{\mathrm{t}}=70 \mathrm{pA}$; (c) schematic models indicate the formation of $1 D$ molecular ribbons based on combination $1+1$. to arrays of ribbons shown in Fig. 2(a) when further annealing the structure at $400 \mathrm{~K}$ to allow molecular free diffusion. Same to Co, both Fe and Ni can form STs with C3PC through metalnitrogen coordinated bonds. Because the BPyB molecule is terminated by $\mathrm{N}$ atoms, we expect that similar surface structures should be obtained by replacing Co with $\mathrm{Fe}$ or $\mathrm{Ni}$ atoms. ${ }^{10,11}$

To investigate the formation mechanism of 1D chains of STs, we repeated the experiments by replacing BPyB molecules by $4^{\prime \prime}$ dicyano-1, $1^{\prime}: 3^{\prime}, 1^{\prime \prime}$-terphenyl (C3PC) molecules with other parameters unchanged. The longer C3PC molecule is terminated by cyano groups. Long-range ordered chains were not obtained at various annealing temperature and molecular coverages. Fig. 4(a) shows a representative STM image of CoC3PC-STs on $\mathrm{Au}(111)$, where the longest chain is marked by a white rectangle. The magnified image (Fig. 4(b)) indicates that the molecular chain is composed of six Co-C3PC-STs. To be noted, two sides of the chain are not straight, as marked by white dashed curves.

The C3PC molecule is slightly longer than BPyB. As a result, the degrees of commensurability between intermolecular lengths in STs and the lattice distance of $\mathrm{Au}(111)$ are different for these two molecules, which might account for their different growth behaviors. To quantitatively elucidate the relation between mismatching degrees and forming structures, $K$-maps with the undermentioned formula was calculated,

$$
K=|1-| m \mathbf{i}+n \mathbf{j}|/ d|
$$

Here, $\mathbf{i}$ and $\mathbf{j}$ represent two basic vectors of $\mathrm{Au}(111)$ as indicated by black arrows in Fig. 5(a) and (b), with $m$ and $n$ as their integer factors. The lattice distances along these two directions are both
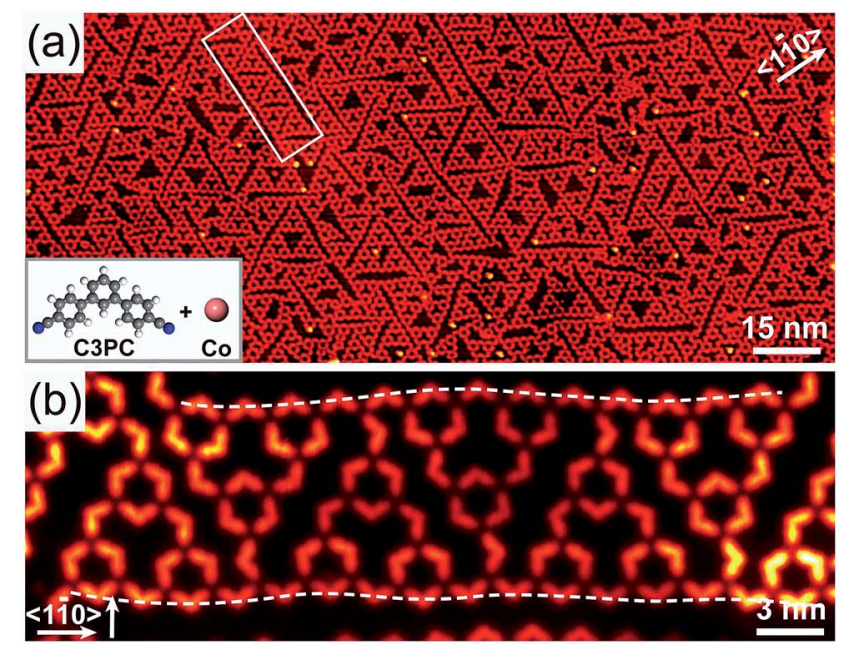

Fig. 4 C3PC-Co-STs formed at high molecular coverage. (a) Overview of irregular network formed by C3PC and Co coordination. The molecular model of C3PC is shown in the left inset. (b) Detailed STM image showing a strip of curved C3PC-Co-ST-2 array indicated by the white rectangle in (a). Both sides of the slightly distorted array are highlighted by dashed curves. Scanning parameters: (a) constantcurrent mode, $V_{\mathrm{B}}=1.8 \mathrm{~V}, I_{\mathrm{t}}=17.5 \mathrm{pA}$; (b) constant-height mode, $V_{\mathrm{B}}=$ $10 \mathrm{mV}, I_{\mathrm{t}}=15.4 \mathrm{pA}$. 
(a)

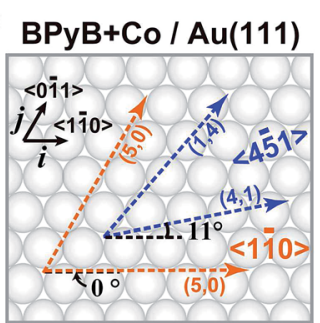

(b)

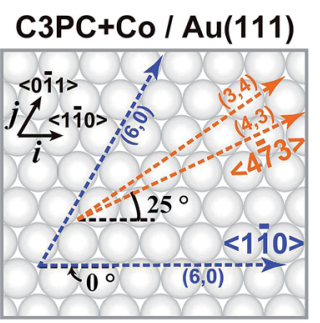

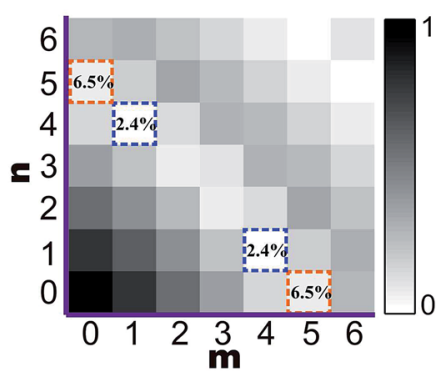

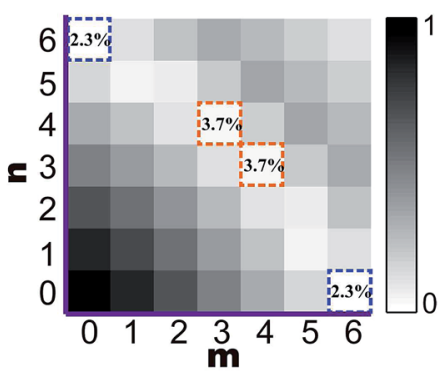

Fig. 5 K-Map of (a) BPyB-Co coordination and (b) C3PC-Co coordination. Each left panel corresponds to surface lattice models of $\mathrm{Au}(111)$ with the best (blue arrows) and second best (orange arrows) matching orientation; each right panel corresponds to relative grayscale $K$ maps.

$0.288 \mathrm{~nm}$. In the formula, $d$ corresponds to the theoretically calculated distance between two neighboring Co atoms, which equals $13.5 \AA$ for Co-BPyB-Co and $16.9 \AA$ for Co-C3PC-Co, respectively. $K$ values at each $(m ; n)$ coordinate, representing the degree of commensurability, were calculated and displayed by squares with different brightness. A smaller $K$ value means a better structural match between STs and $\mathrm{Au}(111)$. Four smallest $K$ values are numbered in each $K$-map to highlight the favorable adsorption direction. For Co-BPyB-STs, the smallest mismatch value is $2.4 \%$ at the coordinate $(4 ; 1)$. This explains well that the sides of Co-BPyB-STs are adsorbed along the $\langle 4 \overline{5} 1\rangle$ directions in measurements. The adsorption energies of $\mathrm{Co}^{-}$ $\mathrm{BPyB}-\mathrm{Co}$ and $\mathrm{Co}-\mathrm{C} 3 \mathrm{PC}-\mathrm{Co}$ motifs at representative coordinates have been calculated to demonstrate the validity of the $K$ map (ESI + ). For Co-BPyB-Co, its adsorption energy at the coordinate of $(4 ; 1)$ is $0.39 \mathrm{eV}$ larger than that at the coordinate of $(5 ; 0)$. The structures with smaller $K$ values are more stable. It is clear that the calculated results are consistent with what the $K$-map indicates. For the longer Co-C3PC-Co motif, the difference of its adsorption energies at the coordinates of $(4 ; 3)$ and $(6 ; 0)$ is only around $0.05 \mathrm{eV}$. The experimentally observed adsorption directions of sides of the STs are along the $\langle 4 \overline{7} 3\rangle$ directions and one side of an ST adsorbs nearly along the reconstruction row. For this kind of adsorption, the structural matching between STs and the herringbone reconstruction of $\mathrm{Au}(111)$ might account for the energy stabilization of STs. Along the $\langle 4 \overline{7} 3\rangle$ directions, the $K$ value is $3.7 \%$, which is rather large and does not favors the formation the long-range ordered structures.

In summary, by using BPyB molecules and Co atoms, onedimensional molecular chains with STs as building blocks were prepared on $\mathrm{Au}(111)$ and studied by low-temperature scanning tunneling microscopy. STs were stabilized by the

coordination interaction between $\mathrm{N}$ atoms of $\mathrm{BPyB}$ and $\mathrm{Co}$ atoms. The chains were only obtained at high molecular coverages and isolated STs up to the fourth order were observed at low coverages. The structure matching between molecular size and surface lattices are attributed to the formation of molecular chains. As a comparison, long-range ordered chains were not obtained for C3PC molecules and Co atoms at various annealing temperature and molecular coverages. To be continued, it is of interest to investigate the surface structures by replacing Co with Fe or the comprehensive result by mixing the two different molecules and novel bicomponent structures are expected to emerge. ${ }^{20}$

\section{Conflicts of interest}

There are no conflicts to declare.

\section{Acknowledgements}

This work was supported by National Natural Science Foundation of China (21522301, 21373020, 21403008, 61321001, 21433011 and 61271050), the Ministry of Science and Technology (2014CB239302 and 2017YFA0205003).

\section{References}

1 R. Sarkar, K. Guo, C. N. Moorefield, M. J. Saunders, C. Wesdemiotis and G. R. Newkome, Angew. Chem., Int. Ed., 2014, 53, 12182-12185.

2 G. R. Newkome, P. Wang, C. N. Moorefield, T. J. Cho, P. P. Mohapatra, S. Li, S.-H. Hwang, O. Lukoyanova, L. Echegoyen, J. A. Palagallo, V. Iancu and S.-W. Hla, Science, 2006, 312, 1782-1785.

3 K. Fujibayashi, R. Hariadi, S. H. Park, E. Winfree and S. Murata, Nano Lett., 2008, 8, 1791-1797.

4 Z. Jiang, Y. Li, M. Wang, D. Liu, J. Yuan, M. Chen, G. R. Newkome, W. Sun, X. Li and P. Wang, Angew. Chem., Int. Ed., 2017, 56, 11450-11455.

5 D. Nieckarz and P. Szabelski, Chem. Commun., 2014, 50, 6843-6845.

6 D. Nieckarz and P. Szabelski, J. Phys. Chem. C, 2013, 117, 11229-11241.

7 J. Shang, Y. Wang, M. Chen, J. Dai, X. Zhou, J. Kuttner, G. Hilt, X. Shao, J. M. Gottfried and K. Wu, Nat. Chem., 2015, 7, 389-393.

8 X. Zhang, N. Li, G.-C. Gu, H. Wang, D. Nieckarz, P. Szabelski, Y. He, Y. Wang, C. Xie, Z.-Y. Shen, J.-T. Lü, H. Tang, L.-M. Peng, S.-M. Hou, K. Wu and Y. Wang, ACS Nano, 2015, 9, 11909-11915.

9 X. Zhang, R. Li, N. Li, G. Gu, Y. Zhang, S. Hou and Y. Wang, Chin. Chem. Lett., 2017, DOI: 10.1016/j.cclet.2017.09.041.

10 Q. Sun, L. Cai, H. Ma, C. Yuan and W. Xu, Chem. Commun., 2015, 51, 14164-14166.

11 N. Li, X. Zhang, G.-C. Gu, H. Wang, D. Nieckarz, P. Szabelski, Y. He, Y. Wang, J.-T. Lü, H. Tang, L.-M. Peng, S.-M. Hou, K. Wu and Y. Wang, Chin. Chem. Lett., 2015, 26, 1198-1202. 
12 X. Zhang, N. Li, L. Liu, G. Gu, C. Li, H. Tang, L. Peng, S. Hou and Y. Wang, Chem. Commun., 2016, 52, 10578-10581.

13 G. Gu, N. Li, L. Liu, X. Zhang, Q. Wu, D. Nieckarz, P. Szabelski, L. Peng, B. K. Teo, S. Hou and Y. Wang, RSC Adv., 2016, 6, 66548-66552.

14 A. Rastgoo-Lahrood, N. Martsinovich, M. Lischka, J. Eichhorn, P. Szabelski, D. Nieckarz, T. Strunskus, K. Das, M. Schmittel, W. M. Heckl and M. Lackinger, ACS Nano, 2016, 10, 10901-10911.

15 A. Wang and M. Zhao, Phys. Chem. Chem. Phys., 2015, 17, 21837-21844.

16 E. van Veen, S. Yuan, M. I. Katsnelson, M. Polini and A. Tomadin, Phys. Rev. B, 2016, 93, 115428.
17 C. Li, X. Zhang, N. Li, Y. Wang, J. Yang, G. Gu, Y. Zhang, S. Hou, L. Peng, K. Wu, D. Nieckarz, P. Szabelski, H. Tang and Y. Wang, J. Am. Chem. Soc., 2017, 139, 13749-13753.

18 N. Li, G. Gu, X. Zhang, D. Song, Y. Zhang, B. K. Teo, L.-M. Peng, S. Hou and Y. Wang, Chem. Commun., 2017, 53, 3469-3472.

19 I. Horcas, R. Fernández, J. M. Gomez-Rodriguez, J. Colchero, J. Gómez-Herrero and A. M. Baro, Rev. Sci. Instrum., 2007, 78, 013705.

20 X. Bouju, C. Mattioli, G. Franc, A. Pujol and A. Gourdon, Chem. Rev., 2017, 117, 1407-1444. 